\title{
Frecuencia de los polimorfismos CYP1A1*2A y deleción del gen GSTM1 en pacientes con carcinoma de células escamosas de laringe en relación al hábito tabáquico: Estudio piloto en Chile
}

\author{
Frequency of CYP1A1*2A and GSTM1 gene polymorphisms in Chilean patients with \\ squamous cell carcinoma of the larynx in relation to smoking habit: a pilot study
}

\author{
Maritza Rahal E¹, María José Herrera J', Luis Quiñones $\mathrm{S}^{2}$, Nancy Farfán $\mathrm{T}^{2}$, Dante Cáceres $\mathrm{L}^{2,3}$, \\ Ángela Roco $\mathrm{A}^{2}$.
}

\begin{abstract}
RESUMEN
Introducción: Entre los factores de riesgo para cáncer laríngeo (CL) son relevantes el consumo de tabaco y alcohol. Estos xenobióticos son metabolizados por un grupo de enzimas, entre las cuales están CYP1A1 y GSTM1, cuyas variantes polimórficas se postulan como factores de riesgo para esta enfermedad.

Objetivos: Describir la frecuencia de las variantes de los polimorfismos de CYP1A1 y GSTM1 en un grupo de pacientes diagnosticados con CL. Analizar la posible correlación entre las variantes genéticas de ambas enzimas y la presencia de CL. Evaluar la influencia del hábito tabáquico en el riesgo de aparición de cáncer escamoso de laringe en pacientes con genotipos de riesgo.

Material y método: Se seleccionaron 35 pacientes con CL entre los años 2000 y 2010 en Servicio de Otorrinolaringología del HBLT y 124 controles reclutados en el Centro de Investigaciones Farmacológicas y Toxicológicas (IFT). A todos los individuos se les registraron datos demográficos y extrajo una muestra de sangre para analizar las variantes polimórficas de CYP1A1 y GSTM1, mediante PCR-RFLP.

Resultados: De un total de 35 pacientes 54,3\% presentan el genotipo GSTM1 (-/-) y 17,1\% el genotipo CYP1A1*2A C/C. En el grupo control $(n=140)$ estas frecuencias fueron de $19,35 \%$ y 10,48\%, respectivamente. Se observó una correlación entre GSTM1 y el CL, estratificado por el hábito tabáquico y alcohólico. No se encontraron relaciones estadísticamente significativas con el hábito alcohólico y/o tabáquico. No se observaron asociaciones entre la patología y la combinación de genotipos o entre genotipos y el hábito tabáquico o alcohólico.

Conclusiones: Los resultados muestran una asociación estadísticamente significativa entre la deleción de GSTM1 (-/-) y el riesgo de presentar CL, lo que refleja el importante papel que juega esta enzima en la desintoxicación de compuestos cancerígenos. Sin embargo, se requiere incrementar el número de pacientes para establecer apropiadamente la relación genético-ambiental que permite adjudicar un papel relevante a estos biomarcadores.

Palabras clave: Cáncer de laringe, polimorfismos genéticos, CYP1A1, GSTM1, metabolismo de xenobióticos.
\end{abstract}

\footnotetext{
Servicio de Otorrinolaringología, Hospital Barros Luco Trudeau (HBLT).

2 Laboratorio de Carcinogénesis Química y Farmacogenética, Centro de Investigaciones Farmacológicas y Toxicológicas (IFT), Facultad de Medicina, Universidad de Chile.

3 División de Epidemiología, Escuela de Salud Pública, Facultad de Medicina, Universidad de Chile.
} 


\section{ABSTRACT}

Introduction: Tobacco and alcohol consumption are recognized risk factors for squamous cell carcinoma of the larynx. These xenobiotics are metabolized by numerous enzymes, among which, CYP1A1 and GSTM1 gene polymorphisms have been identified as risk factors for developing tobacco related cancers as lung and laryngeal carcinomas. Nevertheless, these polymorphisms have not been studied in Chilean patients with squamous cell carcinoma of the larynx.

Aim: To describe, for the first time, the frequency of CYP1A1 and GSTM1 gene polymorphisms in Chilean patients with squamous cell carcinoma of the larynx.

Material and method: We conducted a case-control study. The case group consisted of 35 Chilean patients with squamous cell carcinoma of the larynx; the control group was formed by 124 Chilean subjects without cancer diagnosis. Demographic data as age, sex and quantification of tobacco smoking and alcohol consumption were recorded in all individuals. CYP1A1 and GSTM1 gene polymorphisms were evaluated by polymerase chain reaction and restriction enzymes (PCR-RFLP).

Results: The frequency of CYP1A1*2A C/C genotype was $54,3 \%$ among laryngeal cancer patients and $17,1 \%$ among control subjects. The frequency of GSTM1 (-/-) genotype was 19, $35 \%$ among laryngeal cancer patients and 10, 48\% among control subjects. There were no statistically significant relationships between this gene polymorphisms and tobacco smoking or alcohol consumption. There were no associations between the presence of both gene polymorphisms in the same individual and the presence of laryngeal cancer. Interestingly we found an OR of 8.69 (Cl 2.90 to 26.01) for GSTM1 (-/-) polymorphism and laryngeal cancer, stratified by tobacco smoking and alcohol consumption.

Conclusions: Our work shows that the deletion of GSTM1 could be an important risk factor for squamous cell carcinoma of the larynx in Chilean patients. This finding reflects the important role that detoxification of carcinogenic compounds plays in Chilean population. However, it is necessary to increase the number of studied patients to properly establish the genetic-environmental relationship ascribed to these biomarkers.

Key words: Carcinoma of the larynx, xenobiotics, CYP1A1, GSTM1, gene polymorphisms.

\section{INTRODUCCIÓN}

El cáncer de laringe es la tercera causa de cáncer de cabeza y cuello, constituyendo el $25 \%$ de ellos, siendo el tipo más frecuente el espinocelular, con $95 \%$ del total de cánceres de laringe ${ }^{1}$. Afecta principalmente a hombres, en proporción de 7-10:1 respecto de las mujeres ${ }^{1}$.

Su tratamiento suele ser bastante agresivo dada la consulta tardía que presenta la población chilena ${ }^{1}$. En estos casos, el tratamiento está referido al uso de radioterapia más cirugía, siendo esta última muy mutilante, dando importancia principalmente a la prevención y pesquisa precoz de los pacientes susceptibles. Se ha demostrado que este cáncer presenta una fuerte asociación a consumo de tabaco y en menor medida al alcohol ${ }^{2-4}$. A pesar de esto, no todos los pacientes tabáquicos desarroIlan la patología, por lo que deben existir factores intrínsecos que modulan el efecto final. Dentro de los factores predisponentes a cáncer descritos en la literatura internacional, destacan las enzimas de biotransformación, fundamentales en la desintoxicación de xenobióticos, incluyendo agentes carcinogénicos ${ }^{5,6}$.

Las enzimas de biotransformación constituyen las rutas de metabolización de xenobióticos y se dividen en Fase I y Fase II. La primera corresponde a las reacciones de oxidación, reducción e hidrólisis, aumentando la hidrofilicidad del compuesto con objeto que sea excretado. Si esta fase no es suficiente es posible que el metabolito obtenido de la Fase I ingresa a la Fase II, que corresponde principalmente a conjugaciones con sustancias endógenas como el 
ácido glucurónico, aminoácidos, ácidos grasos, glutatión, grupos metilo, sulfato o acetato, provocando la inactivación de dichos metabolitos y facilitando de esta manera su excreción?.

Dentro de las enzimas de fase I involucradas, destacan la citocromo P450 CYP1A1 y entre las de fase II la glutatión-S-transferasa GSTM1, ambas involucradas en la carcinogénesis asociada a exposición a hidrocarburos aromáticos policíclicos (HAPs- Ej. Benzo(a)pireno), nitrosaminas y dioxinas, tóxicos precancerígenos presentes en el humo del tabaco6,8 (Figura 1). Muchas de estas enzimas son polimórficas, lo que determina diferencias en la metabolización, y por ende, diferencias personales en la susceptibilidad a contraer cáncer de laringe ante la exposición a estos carcinogénicos.

En el ámbito internacional existen diferentes publicaciones que han tratado de correlacionar diversos polimorfismos con cáncer de vía aéreo-digestiva superior, con diversos resultados ${ }^{9-14}$. Sin embargo, estos estudios no son extrapolables a la población nacional dada sus características étnicas ${ }^{15,16}$. Estos ya han sido correlacionados con cáncer en vía aerodigestiva superior, pulmón y próstata en la población chilena ${ }^{15-19}$. Debido a lo anterior, existe la necesidad de estudiar alguna correlación con el cáncer de laringe en la población chilena, dado el alto nivel de tabaquismo existente entre nuestros jóvenes, potenciales portadores de cáncer de laringe en el largo plazo.

Las variantes polimórficas a estudiar corresponden al cambio nucleotídico T3801C en la región 3' de poli-adenilación del gen CYP1A1 que conduce a una variante de mayor expresión de la enzima. Por su parte, la variante de GSTM1 corresponde a una deleción homocigota del gen completo de esta enzima ${ }^{19}$. En los últimos años múltiples estudios, han mostrado la influencia en la susceptibilidad a cáncer escamoso de laringe de los polimorfismos en los genes del citocromo P450, en especial CYP1A124.

\section{OBJETIVO}

Con estos antecedentes se plantea que las variantes polimórficas de las enzimas CYP1A1 y GSTM1, participantes en el proceso de biotransformación de hidrocarburos aromáticos policíclicos (HAPs), compuestos precancerígenos del tabaco, influencian la susceptibilidad a la aparición de cáncer de laringe por exposición a estos compuestos. Dicha hipótesis será abordada mediante la determinación de los diferentes genotipos de los polimorfismos de *2A y -/- de las enzimas CYP1A1 y GSTM1, respectivamente, en pacientes con cáncer de laringe (CL) e individuos control.

\section{MATERIAL Y MÉTODO}

\section{Reactivos y experimentos}

Para la extracción de sangre periférica se utilizaron tubos con EDTA como anticogulante (BD

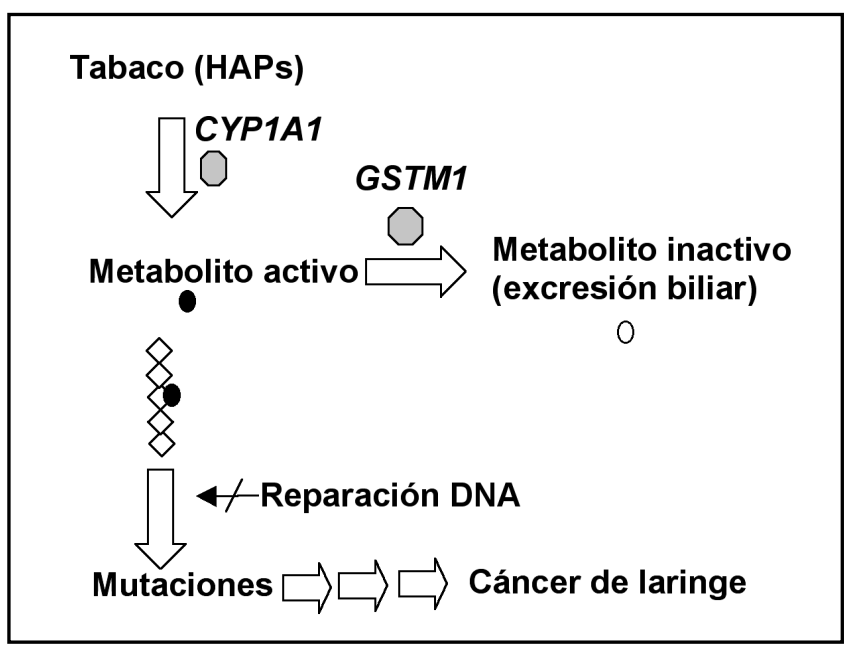

Figura 1. Enzimas de biotransformación y su papel en el metabolismo de hidrocarburos aromáticos policíclicos (HAPs). 
Vacutainer ${ }^{\circledR}$, EE.UU). La determinación de grupos sanguíneos se realizó con un kit comercial (BioClone ${ }^{\circledR}, A B O$ Pharmaceuticals, EE.UU). Para la purificación del ADN se utilizó un sistema comercial de purificación de ADN genómico de sangre periférica (High Pure PCR Template Preparation Kit, Roche Diagnostics GmbH, Mannheim, Alemania). Para las PCRs se utilizaron partidores específicos (sintetizados por Invitrogen, Brasil), $\mathrm{H}_{2} \mathrm{O}$ DPC (Invitrogen, Brasil), dNTPs (mezcla de dATP, dTTP, dGTP y dCTP) (Invitrogen, Brasil), Taq DNA Polymerase Recombinante (Invitrogen, Brasil) con su tampón de reacción 10X (Tris-HCl 200 mM (pH 8), $\mathrm{KCl} 500 \mathrm{mM}$ ), $\mathrm{MgCl}_{2} 500 \mathrm{mM}$ (Invitrogen, Brasil). En el estudio de PCR-RFLP se utilizó la enzima de restricción Mspl (Fermentas, Hanover, EE.UU). Para la visualización de los fragmentos de restricción se utilizaron los marcadores de peso molecular 1001000 pb Hyperladder IV y 1000-10000 pb MassRuler High Range (Bioline, Taunton, MA, EE.UU) en geles de agarosa y poliacrilamida (Bioline, Taunton, MA, EE.UU) con bromuro de etidio (Invitrogen, Brasil). Para la electroforesis de agarosa se utilizó el tampón Tris-borato (TBE) $1 \mathrm{X}$ (Tris 89 mM (Winkler, México), ácido bórico $89 \mathrm{mM}$ (Winkler, México), EDTA 0,5 M pH 8 (Winkler, México) y para la electroforesis vertical se utilizó gel de poliacrilamida (acrilamida 30\% (Winkler, México), Tris HCl 1,5 M pH 8 (Winkler, México), persulfato de amonio (Winkler, México) y TEMED (Merck, Alemania). El tapón de corrida utilizado fue Tris 0,025M (Winkler, México) - Glicina 0,192 M pH 8 (Winkler, México).

Las muestras se procesaron por medio de la técnica de PCR-RFLP. Brevemente, el gen CYP1A1 se amplifica bordeando su sitio Msp1 por medio de Ios partidores C44 y C47, los cuales producen un fragmento de $340 \mathrm{pb}$. El polimorfismo del gen GSTM1 se determinó por medio de los partidores descritos por Ambrosone y cols ${ }^{20}$, cuyo producto es un fragmento de $273 \mathrm{pb}$. El genotipo nulo se determina por medio de la ausencia de este producto en electroforesis en gel de agarosa al $2 \%$.

Digestión por enzimas de restricción (RFLP): Los productos de la amplificación de CYP1A1 se digieren por medio de la enzima de restricción Msp1. Los pacientes positivos para la variante $C$ de este polimorfismo presentan una digestión del producto de la PCR, con lo que se obtiene dos fragmentos; el mayor de $200 \mathrm{pb}$ y el menor de 140 $\mathrm{pb}$, al ser separados por medio de electroforesis en gel de agarosa al $2 \%$.

\section{Diseño del estudio}

El presente trabajo es un estudio caso-control, realizado en el Servicio de Otorrinolaringología del Complejo Asistencial Barros Luco en colaboración con el Laboratorio de Carcinogénesis Química y Farmacogenética (CQF) del Centro de Investigaciones Farmacológicas y Toxicológicas (IFT) de la Facultad de Medicina de la Universidad de Chile.

\section{Pacientes y controles}

Se incluyeron en este estudio de tipo caso-control, pacientes de las siguientes características:

Casos: 35 pacientes adultos atendidos en el Servicio de Otorrinolaringología del Complejo Asistencial Barros Luco, con diagnóstico de cáncer escamoso de laringe con confirmación histológica diagnosticados en los últimos 10 años. Los criterios de exclusión fueron: diagnóstico de cáncer de laringe no escamoso, sospecha de cáncer de laringe no confirmado histológicamente y solicitud explícita del paciente de no participar en este estudio.

Controles: 124 pacientes adultos sin antecedentes de cáncer de laringe reclutados en el Centro de Investigaciones Farmacológicas y Toxicológicas, los cuales debían cumplir con los criterios de exclusión e inclusión de acuerdo los protocolos internos del laboratorio CQF, para el reclutamiento de voluntarios sanos.

Se solicitó a todos los pacientes en estudio, la firma de consentimiento informado para este procedimiento, el cual se realizó estrictamente de acuerdo a lo éticamente aceptado internacionalmente (Protocolo de Helsinki). Este proyecto fue aprobado por el Comité de Ética del Hospital Barros Luco-Trudeau. Adicionalmente, se confeccionó una ficha tipo donde se registraron datos como edad, sexo, edad de inicio de uso de tabaco, frecuencia del hábito tabáquico, índice de tabaco (Brikman) ${ }^{21}$, consumo de alcohol (valor de corte $=280$ y 168 gramos por semana en hombre y mujer, respectivamente), y otras drogas, antecedentes familiares de cáncer de laringe y/o pulmón, patología asociada, antecedentes clínicos: estadio al momento del diagnóstico, conducta biológica del tumor.

Se reclutaron un total de 35 pacientes adultos, con diagnóstico de cáncer escamoso de laringe 
con confirmación histológica y 124 controles: voluntarios adultos sin antecedentes de cáncer de laringe reclutados en el CQF.

A los individuos seleccionados para este estudio se les realizó una extracción de una muestra de sangre periférica para la extracción de ADN de linfocitos periféricos. Esta muestra se almacenó en ácido etilendiaminotetraacético (EDTA) como anticoagulante según los protocolos del CQF hasta su análisis.

Este proyecto contó con el financiamiento de la Sociedad Chilena de Otorrinolaringología, Medicina y Cirugía Cabeza y Cuello.

\section{Determinación del tamaño de muestra y análisis estadístico}

Para el cálculo del tamaño de la muestra se consideró un valor aaa (corresponde al riesgo de cometer un error) de $0,05 \%$ y una potencia estadística de $80 \%$. Para estimar el riesgo de tener CL individuos portadores del gen de riesgo $\mathrm{C}$ y para este estudio se estableció un riesgo de 3,5 veces mayor comparado con los que no poseen en genotipo de riesgo, ello basado en estudios anteriores de nuestro grupo ${ }^{16-19}$. Se consideró una razón caso:control de 1:2, es decir 1 caso por cada 2 controles, lo que da un tamaño de muestra de 46 casos y 61 controles. El tamaño de muestra fue calculado con el software nQuery4.0.
Para la evaluación de la relación entre los polimorfismos genéticos de CYP1A1 y GSTM1, se realizó un estudio tipo caso-control, utilizando los programas Epi Info 6.0 y STATA 5.0. Se analizaron los resultados por medio la prueba de chi cuadrado.

\section{RESULTADOS}

Los datos demográficos y de las variantes genéticas en estudio de los pacientes incluidos se muestran en la Tabla 1. La población estudiada de casos está mayormente formada por hombres y de mayor edad que los controles. Como era de esperar el hábito tabáquico es mayor en los casos que en los controles, encontrándose que más del $90 \%(92,6 \%)$ de los casos son consumidores de tabaco, a diferencia de los controles en donde el hábito tabáquico es menor al 50\% (35,4\%). No se observan diferencias en el hábito alcohólico entre los dos grupos. Respecto de las variables genéticas a estudiar, se observa que la frecuencia del alelo mutado de CYP1A1 ( $\left.{ }^{*} 2 \mathrm{~A}\right)$ es levemente mayor en los casos así como también la frecuencia de la deleción homocigota de GSTM1 (genotipo nulo), sin asociación estadísticamente significativa. Un patrón genotípico representativo se muestra en la Figura 2, en donde es posible observar las bandas

Tabla 1. Distribución de las variables estudiadas en los grupos casos y control

\begin{tabular}{|lcc|}
\hline & Controles & Casos \\
\hline Hombres & $54 \%$ & $88,60 \%$ \\
Edad & $50,4 \pm 10,2$ & $63,7 \pm 7,3$ \\
Tabaquismo (paq/año) & & \\
0 & $64,6 \%$ & $7,4 \%$ \\
$<20$ & $35,4 \%$ & $24,1 \%$ \\
$>20$ & $0 \%$ & $68,52 \%$ \\
Alcohol & $21,77 \%$ & $25,70 \%$ \\
Frecuencia alélica CYP1A1*2AWT & 0,63 & 0,61 \\
${ }^{2 A}$ & 0,37 & 0,39 \\
Frecuencia GSTM1*0 & & \\
Presente & 0,64 & 0,57 \\
Nulo & 0,36 & 0,43 \\
\hline
\end{tabular}

WT= Alelo "wild type" o silvestre.

${ }^{*} 2 \mathrm{~A}=$ Alelo mutado $(\mathrm{T} / \mathrm{C})$

Presente $=$ gen GSTM1 presente (activo)

Nulo = Deleción homocigota de GSTM1 (del/del) 


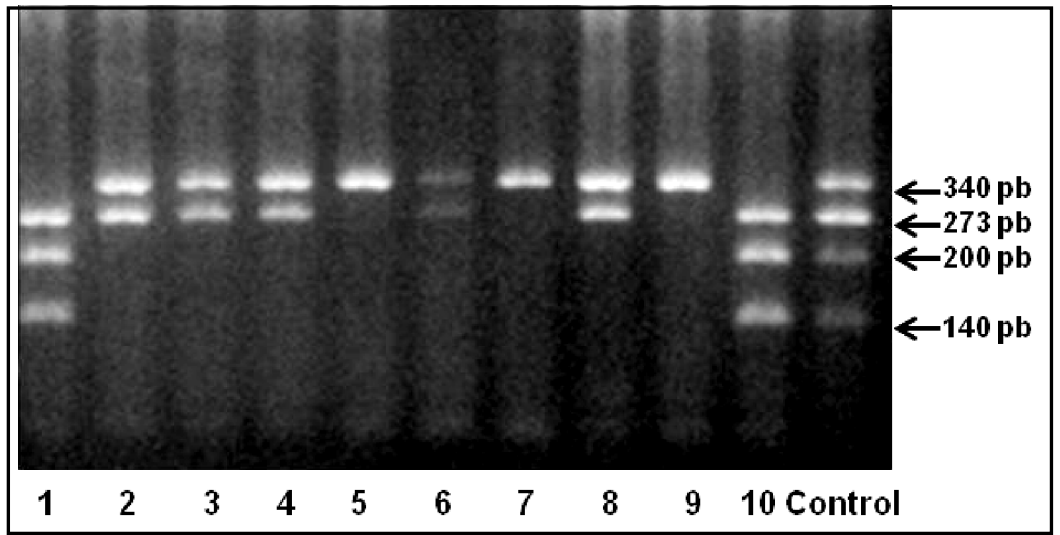

Figura 2. Patrones electroforéticos de la genotipificación de 10 pacientes con cáncer de laringe.

correspondientes a la presencia del gen GSTM1 (273 $\mathrm{pb})$, las bandas correspondientes al fragmento de amplificación de CYP1A1 (340 pb) y sus bandas de digestión (200 pb y 140 pb) luego de la digestión con la enzima de restricción Msp1, lo que denota la presencia de la variante ${ }^{*} 2 \mathrm{~A}$. El control muestra la presencia de todas las bandas.

La Tabla 2 muestra un estudio de asociación entre la presencia de las variantes genéticas de CYP1A1 y el riesgo de cáncer de laringe en relación al hábito tabáquico. En la tabla se puede apreciar que en individuos con genotipo silvestre (WTMT) el hábito tabáquico se asocia con el riesgo de presentar la enfermedad de manera dosis-dependiente. Así mismo, es posible observar que la presencia de la variante mutada ( $\left.{ }^{\star} 2 A\right)$ muestra la misma tendencia, con valores de riesgo mayor, aunque no significativos estadísticamente, indicando un efecto modificador de la variante sobre el riesgo inducido por el hábito tabáquico.

La Tabla 3 muestra el estudio de asociación entre la deleción homocigota de GSTM1 y el riesgo de cáncer de laringe, en relación con el hábito tabáquico. En la tabla es posible apreciar que en individuos con genotipo silvestre (GSTM1 presente), el hábito tabáquico se asocia con el riesgo de presentar la enfermedad de manera dosis-dependiente. Igual que para el caso del alelo mutado de CYP1A1, la presencia de la variante mutada (nula) muestra la misma tendencia, con valores de riesgo mayor, indicando un efecto modificador de la variante sobre el riesgo inducido por el hábito tabáquico.

Tabla 2. Asociación entre genotipos de CYP1A1 y hábito tabáquico

\begin{tabular}{|lcccccc|}
\hline $\begin{array}{l}\text { Genotipo } \\
\text { CYP 1 A 1 }\end{array}$ & $\begin{array}{c}\text { Hábito } \\
\text { tabáquico } \\
\text { (PAQ/año) }\end{array}$ & Casos & Controles & ORa & IC95\% & p-value \\
\hline WT/WT & 0 & $2,0 \%$ & $27,1 \%$ & 1 & - & \\
WT/WT & $<20$ & $9,8 \%$ & $16,7 \%$ & 0,13 & $0,85-192,4$ & 0,08 \\
WT/WT & $>20$ & $25,5 \%$ & $0 \%$ & $\infty$ & - & $<0,0001$ \\
WT/*2A & 0 & $5,9 \%$ & $25,0 \%$ & 3,25 & $0,29-84,01$ & 0,5828 \\
WT/*2A & $<20$ & $9,8 \%$ & $13,2 \%$ & 10,40 & $1,07-248,30$ & 0,0397 \\
WT/*2A & $>20$ & $31,4 \%$ & 0 & $\infty$ & - & $<0,0001$ \\
${ }^{*} 2 \mathrm{~A} /{ }^{*} 2 \mathrm{~A}$ & 0 & $0 \%$ & $24,0 \%$ & 0 & - & - \\
${ }^{*} 2 \mathrm{~A} /{ }^{*} 2 \mathrm{~A}$ & $<20$ & $3,9 \%$ & $5,7 \%$ & 9,45 & $0,59-291,48$ & 0,1760 \\
${ }^{*} 2 \mathrm{~A} /{ }^{*} 2 \mathrm{~A}$ & $>20$ & $11,8 \%$ & 0 & $\infty$ & - & $<0,0001$ \\
\hline
\end{tabular}

WT= Alelo "wild type" 0 silvestre

${ }^{\star} 2 \mathrm{~A}=$ Alelo mutado $(\mathrm{T} / \mathrm{C})$

ORa: Odds Ratio ajustada por edad y sexo 
Tabla 3. Asociación entre genotipos GSTM1 y hábito tabáquico

\begin{tabular}{|lcccccr|}
\hline $\begin{array}{l}\text { Genotipo } \\
\text { GSTM1 }\end{array}$ & $\begin{array}{c}\text { Hábito } \\
\text { tabáquico } \\
\text { (PAQ/año) }\end{array}$ & Casos & Controles & ORa & IC 95\% & p-value \\
\hline Presente & 0 & $5,6 \%$ & $40,1 \%$ & 1 & & \\
Presente & $<20$ & $11,1 \%$ & $23,4 \%$ & 0,29 & $0,71-18,31$ & 0,1573 \\
Presente & $>20$ & $38,9 \%$ & $0 \%$ & $\infty$ & - & $<0,0001$ \\
Nulo & 0 & $1,9 \%$ & $24,5 \%$ & 0,55 & $0,02-6,16$ & 1,0000 \\
Nulo & $<20$ & $13,0 \%$ & $12,0 \%$ & 7,81 & $1,63-42,04$ & $<0,0049$ \\
Nulo & $>20$ & $29,6 \%$ & $0 \%$ & $\infty$ & - & $<0,0001$ \\
\hline
\end{tabular}

Presente $=$ gen GSTM1 presente (activo)

Nulo = Deleción homocigota de GSTM1 (del/del)

ORa: Odds Ratio ajustada por edad y sexo

La Tabla 4 muestra un estudio de asociación entre la presencia de genotipos combinados de CYP1A1 y GSTM1, y el riesgo de cáncer de laringe, en relación con el hábito tabáquico. En esta tabla es posible apreciar que para individuos con genotipo silvestre de ambas enzimas el hábito tabáquico se asocia con el riesgo de presentar la enfermedad de manera dosisdependiente, sin embargo, cuando se analiza este riesgo considerando la presencia de una 0 dos variantes mutadas de CYP1A1 y GSTM1, de manera heterocigota u homocigota el riesgo se ve aumentado, siendo el riesgo mayor para la asociación de las variantes mutadas de ambas enzimas y el consumo de más de 20 paquetes de cigarrillos al año.

\section{DISCUSIÓN}

Tal y como se muestra en la Figura 1, las enzimas de biotransformación de Fase I, CYP1A1 y de Fase II,

Tabla 4. Asociación entre genotipos combinados (CYP1A1 y GSTM1) y hábito tabáquico

\begin{tabular}{|c|c|c|c|c|c|c|c|}
\hline $\begin{array}{l}\text { Genotipo } \\
\text { GSTM1 }\end{array}$ & $\begin{array}{l}\text { Genotipo } \\
\text { CYP1a1 }\end{array}$ & $\begin{array}{c}\text { Hábito } \\
\text { tabáquico } \\
\text { (Paq 20) }\end{array}$ & Casos & Controles & Ora & Ic95\% & $\mathrm{P}$-value \\
\hline Presente & Wt/Wt & 0 & $2,0 \%$ & $16,7 \%$ & 1 & - & - \\
\hline Presente & Wt/Wt & $<20$ & $3,9 \%$ & $9,9 \%$ & 3,37 & $0,22-101,15$ & 0,6845 \\
\hline Presente & Wt/Wt & $>20$ & $13,7 \%$ & $0 \%$ & $\infty$ & - & $<0,0001$ \\
\hline Presente & $\mathrm{Wt} /{ }^{*} 2 \mathrm{a}$ & 0 & $3,9 \%$ & $14,1 \%$ & 2,37 & $0,15-70,14$ & 0,9086 \\
\hline Presente & $\mathrm{Wt} /{ }^{\star} 2 \mathrm{a}$ & $<20$ & $5,9 \%$ & $8,9 \%$ & 5,65 & $0,46-152,87$ & 0,2829 \\
\hline Presente & $\mathrm{Wt} /{ }^{*} 2 \mathrm{a}$ & $>20$ & $19,6 \%$ & $0 \%$ & $\infty$ & - & $<0,0001$ \\
\hline Presente & ${ }^{*} 2 \mathrm{a} /{ }^{*} 2 \mathrm{a}$ & 0 & 0 & $9,4 \%$ & 0 & - & - \\
\hline Presente & ${ }^{*} 2 \mathrm{a} /{ }^{*} 2 \mathrm{a}$ & $<20$ & $2,0 \%$ & $4,7 \%$ & 3,56 & $0,0-148,19$ & 0,9523 \\
\hline Presente & ${ }^{*} 2 \mathrm{a} /{ }^{*} 2 \mathrm{a}$ & $>20$ & $5,9 \%$ & $0 \%$ & $\infty$ & - & $<0,0001$ \\
\hline Nulo & $\mathrm{Wt} / \mathrm{Wt}$ & 0 & $0 \%$ & $10,4 \%$ & 0 & - & - \\
\hline Nulo & Wt/Wt & $<20$ & $5,9 \%$ & $6,8 \%$ & 7,38 & $0,58-203,43$ & 0,1840 \\
\hline Nulo & Wt/Wt & $>20$ & $11,8 \%$ & $0 \%$ & $\infty$ & - & $<0,0001$ \\
\hline Nulo & $\mathrm{Wt} /{ }^{*} 2 \mathrm{a}$ & 0 & $2,0 \%$ & $10,9 \%$ & 1,52 & $0,00-59,71$ & 0,6592 \\
\hline Nulo & $\mathrm{Wt} /{ }^{\star} 2 \mathrm{a}$ & $<20$ & $3,9 \%$ & $4,2 \%$ & 8,00 & $0,47-257,91$ & 0,2555 \\
\hline Nulo & $\mathrm{Wt} /{ }^{*} 2 \mathrm{a}$ & $>20$ & $11,8 \%$ & $0 \%$ & $\infty$ & - & $<0,0001$ \\
\hline Nulo & ${ }^{*} 2 \mathrm{a} /{ }^{*} 2 \mathrm{a}$ & 0 & 0 & $3,1 \%$ & 0 & - & - \\
\hline Nulo & ${ }^{*} 2 \mathrm{a} /{ }^{*} 2 \mathrm{a}$ & $<20$ & $2,0 \%$ & $1,0 \%$ & 16,00 & $0,0-1089,68$ & 0,3802 \\
\hline Nulo & ${ }^{\star} 2 \mathrm{a} /{ }^{*} 2 \mathrm{a}$ & $>20$ & $5,9 \%$ & $0 \%$ & $\infty$ & - & $<0,0001$ \\
\hline
\end{tabular}

$\mathrm{WT}=$ Alelo "wild type" o silvestre.

${ }^{\star} 2 \mathrm{~A}=$ Alelo mutado $(\mathrm{T} / \mathrm{C})$

Presente = gen GSTM1 presente (activo)

Nulo = Deleción homocigota de GSTM1 (del/del)

ORa: Odds Ratio ajustada por edad y sexo 
GSTM1 metabolizan de manera secuencial los agentes precancerígenos del tabaco, tales como los hidrocarburos policíclicos aromáticos (HAPs), siendo la enzima CYP1A1 responsable de la formación del metabolito activo, y la enzima GSTM1 la responsable de su eliminación biliar. Cuando la enzima GSTM1 no es capaz de excretar el metabolito activo de HAPs se produce un aducto en el ADN, el que una vez que actúa el sistema de reparación puede generar una mutación, dando inicio a un proceso tumoral.

El gen CYP1A1 completo ha sido secuenciado y se ha informado de la existencia de varios alelos. De éstos, cuatro son los más estudiados: CYP1A1*2A, CYP $1 A 1{ }^{*} 2 \mathrm{C}, \mathrm{CYP} 1 \mathrm{~A} 1{ }^{*} 3$, específico de las poblaciones de origen africano; y CYP1 $11^{*} 4$, encontrado en poblaciones alemanas, polacas y turcas ${ }^{16}$.

En estudios anteriormente realizados por nuestro grupo de investigación, encontramos una relación en subgrupos de la población chilena, mostrando que la etnia juega un papel importante no sólo en la frecuencia de los alelos, sino también en la interacción entre ellos ${ }^{16}$. Por otro lado, la importancia de la variante ${ }^{\star} 2 \mathrm{C}$ (ile/val o Ncol) de CYP1A1 ha sido considerada menor, puesto que al parecer, no afecta significativamente la actividad catalítica de la enzima CYP1A1 15-17.

Por otro lado, la glutatión S-transferasa humana es una familia de multigenes de enzimas diméricas solubles, entre las que se incluye GSTM1, responsable en gran medida de la desintoxicación de HAPs mediante conjugación con glutatión. Esta enzima tiene una actividad deficiente en aproximadamente $50 \%$ de la población caucásica y el $20 \%$ de población chilena mestiza ${ }^{15}$. Se ha postulado que la frecuencia de la deleción estaría aumentada en pacientes con cáncer ${ }^{15-19}$.

De acuerdo a lo anterior, en el presente trabajo se estudió los genotipos de los polimorfismos CYP1A1*2A y GSTM1nulo en 35 casos chilenos con cáncer de laringe y 124 individuos control de la población general.

Los resultados obtenidos muestran una mayor frecuencia del genotipo nulo de GSTM1 y de la variante *2A de CYP1A1 en casos que en controles, sin asociación estadística (Tabla 1). Del mismo modo, el hábito tabáquico es considerablemente mayor en casos que en controles.

Cuando se analiza la influencia del genotipo de estas dos enzimas en el riesgo de cáncer de laringe, con respecto al hábito tabáquico es posible observar que en presencia de las variantes mutadas 0 variantes "de riesgo" ( ${ }^{\star} 2 \mathrm{~A}$ y nula) existe una clara influencia de estas variantes en el riesgo de presentar la patología (Tablas 2 y 3) lo que no se observa en ausencia del hábito tabáquico. Esto es perfectamente esperable debido a que aun cuando los individuos presenten las variantes genéticas "de riesgo" si no se exponen al/los agente/s precancerígenos no existirá el riesgo de iniciar un cáncer, lo que ratifica el origen genético-ambiental de esta patología.

Cuando se analizaron los genotipos combinados de CYP1A1 y GSTM1 en asociación con el hábito tabáquico como factores de riesgo de cáncer de laringe fue posible observar que el planteamiento anterior fue ratificado, demostrándose que en presencia de la variante de riesgo de ambas enzimas en un mismo individuo, de manera heterocigota u homogicota el riesgo de presentar la patología es aún mayor (Tabla 4).

A pesar de esos hallazgos cabe destacar que un gran porcentaje de las asociaciones no alcanzaron nivel de significancia, lo que se debe al bajo número de pacientes con respecto al número de controles, lo que se agudiza aún más en las asociaciones de genotipos que disminuye aún más del número de individuos por grupo de análisis, llegando a ser " 0 " en algunos grupos. Esta situación se observó generalmente cuando se definieron grupos que presentaran las mutaciones y al mismo tiempo fumaran más de 20 paquetes de cigarrillos al año en el grupo control, lo que sí se podía observar en el grupo de casos. Es decir, los tres factores de riesgo, $\operatorname{CYP} 1 \mathrm{~A} 1{ }^{*} 2 \mathrm{~A}$, GSTM1 nulo (genéticos) y hábito tabáquico (ambiental) se concentraban en los pacientes.

En trabajos previos realizados por nuestro grupo, hemos reportado asociaciones estadísticamente significativas entre el alelo $\mathrm{C}$ de CYP1A1 y otros cánceres $^{18-20}$, sin embargo, en el presente estudio, a pesar que el alelo *2A del polimorfismo CYP1A1*2A y el genotipo nulo de GSTM1 se encuentran aumentados en los casos, no se obtiene diferencia estadísticamente significativa cuando se analizan los genes independientemente del hábito tabáquico, y sólo se logra establecer significancia en algunas asociaciones de estas variantes con el hábito tabáquico, evaluado a través del índice de Brikman ${ }^{21}$.

Investigaciones recientes de otros autores muestran resultados controversiales, algunos han propuesto que no existe asociación entre CYP1A1 *2A y cáncer de laringe ${ }^{22}$ y otros soportan nuestra hipótesis proponien- 
do una asociación entre estas dos variantes, y otras, con cáncer de cabeza y cuello23. Al respecto un reciente metaanálisis realizado por Zhuo et al24 sugiere una asociación de estas variantes con cáncer de laringe, pero de una manera etnia-dependiente ${ }^{24}$, sustentando nuestra idea que la población chilena, por considerarse una etnia mixta, posee características particulares que requieren una descripción y evaluación del papel de los polimorfismos genéticos de estas enzimas en esta y otras patologías.

La alta frecuencia observada del alelo * $2 \mathrm{~A}$ y del genotipo nulo de GSTM1 tanto en pacientes como en los controles sugiere que los individuos chilenos podrían ser más susceptibles a los cánceres originados por compuestos cancerígenos del tabaco que los individuos caucásicos, asiáticos 0 afroamericanos, aun cuando esta susceptibilidad depende además de la presencia relativa de varias enzimas polimórficas, incluyendo aquellas que no pertenecen a las enzimas de biotransformación de Fase I y/o Fase II en cada etnia.

Los resultados presentados en este trabajo constituyen un estudio piloto que seguirá siendo ampliado en el futuro inmediato con objeto de ratificar el papel propuesto para estas variantes polimórficas en cáncer de laringe. La estrategia a abordar consiste en aumentar el número de casos y eventualmente controles para obtener una relación caso:control de 1:2 y de esta manera asignar un poder estadístico mayor al estudio.

Por otro lado, el papel de estas enzimas en la metabolización HAPs en tejido tumoral queda por ser dilucidado, de modo que adicionalmente se establezca la magnitud de la formación de metabolitos activos in situ en el tejido afectado.

Sin perjuicio de 10 anterior, estos resultados preliminares constituyen la primera aproximación a la evaluación de las enzimas de biotransformación de cancerígenos de humo de cigarrillo como biomarcadores de susceptibilidad a cáncer de laringe.

\section{CONCLUSIONES}

Este estudio piloto sugiere que la variante alélica * $2 \mathrm{~A}$ de CYP1A1 y el genotipo nulo de GSTM1 modifican el riesgo de presentar cáncer de laringe provocado por el hábito tabáquico.

Esta hipótesis debe ser validada en un estudio con mayor número de pacientes de modo de alcanzar un mayor poder estadístico.
La descripción del riesgo de presentar cáncer de laringe en individuos fumadores que presenten la variante alélica *2A de CYP1A1 y el genotipo nulo de GSTM1 debe considerar la descripción étnica de los grupos de estudio, especialmente en poblaciones mestizas como la chilena.

Agradecimientos: Los autores desean agradecer la labor técnica realizada por la Srta. Paula Santander quien realizó parte de las genotipificaciones de este trabajo.

Fuente de financiamiento: Este trabajo fue financiado por el Concurso de Investigación de la Sociedad Chilena de Otorrinolaringología, Medicina y Cirugía Cabeza y Cuello.

\section{BIBLIOGRAFÍA}

1. Pradenas M, Vallejos MP, Rahal M, Cisternas M. Cáncer de laringe e hipofaringe. Experiencia del Hospital Barros Luco Trudeau 1990-2001. Rev Otorrinolaringol Cir Cabeza Cuello 2004; 64: 1320.

2. LEE KW, Kuo WR, TSAI SM, y CoL. Different impact from betel quid, alcohol and cigarette: risk factors for pharyngeal and laryngeal cancer. Int $J$ Cancer 2005; 117(5): 831-6.

3. Menvielle G, Luce D, Goldberg P, Bugel I, Leclerc A. Smoking, alcohol drinking and cancer risk for various sites of the larynx and hypopharynx. A case-control study in France. Eur $J$ Cancer Prev 2004; 13(3): 165-72.

4. Talamini R, Bosetti C, La Vecchia C, y col. Combined effect of tobacco and alcohol on laryngeal cancer risk: a case-control study. Cancer Causes Control 2002; 13(10): 957-64.

5. Brockmoller J, Cascorbi I, Kerb R, Sachse C, Roots I. Polymorphisms in xenobiotic conjugation and disease predisposition. Toxicol Lett 1998; 102103: 173-83.

6. Raunio H, Husgafvel-Pursiainen K, Anttila S, Hietanen E, Hirvonen A, Pelkonen 0. Diagnosis of polymorphisms in carcinogen-activating and inactivating enzymes and cancer susceptibilitya review. Gene 1995; 159(1): 113-21.

7. IOANnides C. Enzyme Systems that Metabolise Drugs and Other Xenobiotics. Health (San Francisco) 4, 0-471 (2001). 
8. Gajecka M, Rydzanicz M, Jaskula-Sztul R, Kujawski M, Szyfter W, Szyfter K. CYP1A1, CYP2D6, CYP2E1, NAT2, GSTM1 and GSTT1 polymorphisms or their combinations are associated with the increased risk of the laryngeal squamous cell carcinoma. Mutation Research 2005; 574: 112-23.

9. Park J, Muscat J, Ren Q, y col. CYP1A1 and GSTM1polimorphism and oral cancer risk. Cancer Epidemiol Biomarkers Prev 1997; 6(10): 791-7.

10. Olshan AF, Weissler MC, Watson MA, Bell DA. GSTM1, GSTT1, CYP1A1 and NAT1 polymorphism, tobacco use and the risk of head and neck cancer. Cancer Epidemiol Biomarkers Prev 2000; 9(2): 185-91.

11. Matthias C, Bockmuhl U, Jahnke $V$, y col. Polymorphism in cytochrome P450 CYP2D6, CYP1A1, CYP2E6 and glutathione S-transferasa and susceptibility to tobacco-related cancers: studies in upper aerodigestive tract cancers. Pharmacogenetics 1998; 8(2): 91-100.

12. Matthias C, Bockmuhl U, Jahnke V, y col. The glutathione S-transferase GSTP1 polymorfism: effects on susceptibility to oral/pharingeal and laryngeal carcinomas. Pharmacogenetics 1998; 8(1): 1-6.

13. Jahnke V, Strange R, Matthias C, Fryer A. Glutathione S-transferase and cytochrome P450 as risk factors for laryngeal carcinoma. Eur Arch Otorhinolaryngol 1997; 254 Suppl 1: s147-9.

14. Jahnke V, Matthias C, Strange R. Glutathione Stransferase and citochrome P450 polymorphism as risk factors for squamous cell carcinoma of the larynx. Am J Surg 1996; 172(6): 671-3.

15. Quiñones L, Berthou F, Varela N, Simon B, Gil L, LUCAS $D$. Ethnic susceptibility to lung cancer: differences in CYP2E1, CYP1A1 and GSTM1 genetic polymorphisms between French Caucasian and Chilean populations. Cancer Letters 1999; 141 (1-2): 167-71.

16. Quiñones L, Lee K, Varela N, y col. Farmacogenética del cáncer: Estudio de variaciones genéticamente determinadas en la susceptibilidad a cáncer por exposición a xenobióticos. Rev Med Chil 2006; 134 (4): 499515.

17. QuiÑones L, LuCAS D, Godoy J, y COL. CYP1A1, CYP2E1 and GSTM1 genetic polymorphisms. The effect of single and combined genotypes on lung cancer susceptibility in chilean people. Cancer Letters 2001; 174(1): 35-44

18. Acevedo C, Opazo JL, Huidobro C, Cabezas J, IturRieta J, Quiñones L. Positive Correlation Between single or combined genotypes of CYP1A1 and GSTM1 in relation to prostate cancer in chilean people. The Prostate 2003; 57(2): 111-7.

19. Cordero K, Espinoza I, Caceres D, y col. Oral cancer susceptibility associated with CYP1A1 and GSTM1 genotypes in Chilean people. Oncology Letters 2010; 1(3): 549-54.

20. Lucas D, Menez C, Floch F, y col. Cytochrome P4502E1 and P4501A1 genotypes and susceptibility to cirrhosis or upper digestive tract cancer in alcoholic caucasians. Alcohol Clin Exp Res 1996; 20: 1033-7.

21. BRIKMAn GL and Coates 0 . The effect of bronchitis, smoking and occupation in ventilation. Ann Rev Respir Dis 1963; 87: 68-93.

22. Cury NM, Russo A, Galbiatti AL, Ruiz Mt, Raposo LS, Maniglia JV, Pavarino EC, Goloni-Bertollo EM. Polymorphisms of the CYP1A1 and CYP2E1 genes in head and neck squamous cell carcinoma risk. Mol Biol Rep 2012; 39(2): 1055-63.

23. Sharma R, Ahuja M, Panda NK, Khullar M. Interactions among genetic variants in tobacco metabolizing genes and smoking are associated with head and neck cancer susceptibility in North Indians. DNA Cell Biol 2011; 30(8): 611-6.

24. Zhuo WL, Wang Y, Zhuo XL, Zhu B, Zhu Y, Chen ZT. Polymorphisms of CYP1A1 and GSTM1 and laryngeal cancer risk: evidence-based metaanalyses. J Cancer Res Clin Oncol2009; 135(8): 1081-90.

\author{
Dirección: María José Herrera J. \\ Hospital Barros Luco Trudeau \\ Gran Avenida José Miguel Carrera 3204, San Miguel, Santiago \\ E mail: ma.jose.hj@gmail.com
}

